\title{
FORMATION WATER RESISTIVITY $\left(\mathrm{R}_{\mathrm{W}}\right)$ DETERMINATION: THE SP METHOD
}

\author{
USHIE, FA
}

\author{
Department of Geology, Faculty of Science, PMB 5323, University of Port Harcourt, Port Harcourt, Nigeria.
}

\begin{abstract}
Formation water resistivity represents the resistivity value of the water (uncontaminated by drilling mud) that saturates the porous formation. It is also referred to as connate water or interstitial water. Its resistivity can be determined by a number of methods, one of which is by the SP curve discussed in this work. Analysis of wire-line log data depends on the assumption that the only conductive medium in a formation is the pure water which supplies the energy and drive in reservoirs. So, physical properties of this formation water can be determined, one of which is its electrical resistivity and this eventually leads to water saturation determination - an important aspect of reservoir evaluation. This paper presents a review and comparative assessment of the graphical, vis-à-vis the calculative means of $R_{w}$ determination by the SP method. @ JASEM
\end{abstract}

Many of today's oil reservoirs are composed of sediments, which were once deposited in Marine, deltaic and other aquatic environments. Consequently, these sedimentary beds were originally saturated by salt water. Part of this water was displaced in the process of diagenesis and oil accumulations, the other remains, suspending the hydrocarbons because of their density contrast. That which remains generally is known as "Connate" or "Interstitial" water because the water was "born with" and is stored in the interstices of the sediments.

Schlumberger (1989) defined formation water as the water uncontaminated by drilling mud that saturates the formation rock. Analysis of wire line log data depends on the assumption that the only conductive medium present in the formation is the pore water; the matrix and hydrocarbons are nonconductive. Physical properties of this formation water can be determined, one of which is electrical resistivity. Formation water is the free water which supplies the energy for the water drive in reservoirs; and its resistivity is variable depending on the salinity, temperature and whether or not the formation contains hydrocarbons. At a given salinity, the higher the temperature the lower the resistivity, and the water resistivity at any formation temperature, can be calculated from the water resistivity at another formation temperature, knowing both the temperature and temperature offsets using this formula:

$\mathrm{R}_{\mathrm{w}}$ at $\mathrm{FT}_{2} \quad=\mathrm{R}_{\mathrm{w}} \mathrm{FT}_{1},\left(\mathrm{FT}_{1}+\mathrm{C}\right)\left(\mathrm{FT}_{2}+\mathrm{C}\right)$.

Where $\mathrm{FT}_{1}=$ Initial formation temperature

$\mathrm{FT}_{2} \quad=$ Formation Temperature for which $\mathrm{R}_{\mathrm{w}}$ is being determined.

$\mathrm{C}=21.5$ for Temperature in ${ }^{\circ} \mathrm{C}$

(Smolen, 1977).

It has also been established (Schlumberger, 1989) that the water resistivity determined from a hydrocarbon-bearing zone is usually greater than that from the zone bearing only formation water. Determination of formation water resistivity is very important in calculating water and/or hydrocarbon saturation, in the determination of salinity if temperature is known and in understanding the variations of resistivity from the well wall into the formation by comparing it with the resistivity of the mud filtrate. In both SP and $\mathrm{R}_{\mathrm{wa}}$ comparison methods, wire-line logs provide all the needed parameters to determine the formation water resistivity.

\section{THE SP METHOD}

In many cases, a good value of formation water resistivity $R_{w}$ can easily be found by the SP curve read in clean (non-shale) formations because the SP can be used to distinguish lithology such as shaly from sandy formations. The static SP (SSP) value in a clean formation is related to the chemical activities $\left(a_{w}\right.$ and $\left.a_{m f}\right)$ of the formation water through the formula:

$$
\mathrm{SSP}=\mathrm{K} \log a_{w} / a_{m f}
$$

Where $\mathrm{K}=$ Constant and varies in direct proportion with temperature especially in $\mathrm{NaCl}$ solutions

$$
\begin{aligned}
& \mathrm{K}=61+0.133 \mathrm{~T} \text { in }{ }^{\circ} \mathrm{F} \\
& \mathrm{K}=65+0.24 \mathrm{~T} \text { in }{ }^{\circ} \mathrm{C} \\
& a_{w}=\text { Chemical activity of water } \\
& a_{m f}=\text { Chemical activities of mud filtrate. }
\end{aligned}
$$

For pure Nacl solutions that are not too concentrated, resistivities are inversely proportional to activities.

Therefore,

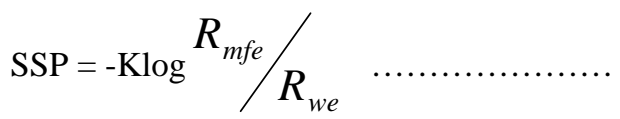


Where $\mathrm{R}_{\mathrm{we}}=0.075 / \mathrm{S}_{\mathrm{w}}$ at $77^{\circ} \mathrm{F}\left(25^{\circ} \mathrm{C}\right)$ and is the equivalent formation water resistivity; and $\mathrm{R}_{\mathrm{mfe}}=$ equivalent mud filtrate resistivity.

After we have been able to relate these resistivities to the SP value for a particular zone, we would then follow the procedure below in determining the formation water resistivity $\left(\mathrm{R}_{\mathrm{w}}\right)$ using the SP method.

1. Establish the shale baseline on the SP curve.

2. Pick out clean permeable zones.

3. Do all the thick zones have about the same SP value? If yes, then pick any thick zone, but otherwise, pick thick zone near and/or the zone you are interested in.

4. Determine the formation temperature i.e. the temperature of this zone chosen, using surface temperature, the bottom hole temperature and the total depth with the formula:

$$
T_{f}=\left(T_{T D}-T_{0}\right) \frac{D_{f}}{T_{D}}+T_{0}
$$

Where $\mathrm{T}_{\mathrm{f}}=$ Temperature of the formation in ${ }^{\circ} \mathrm{F}$ or ${ }^{\circ} \mathrm{C}$.

$\mathrm{T}_{\mathrm{TD}}=$ Temperature at total depth (Bottom hole Temp.) in ${ }^{\circ} \mathrm{F}$ or ${ }^{\circ} \mathrm{C}$.

$\mathrm{T}_{0} \quad=$ Mean surface temperature (in ${ }^{\circ} \mathrm{F}$ or ${ }^{\circ} \mathrm{C}$ ).

$\mathrm{D}_{\mathrm{f}} \quad=$ Depth to formation (in $f t$ or $m$ ).

$\mathrm{T}_{\mathrm{D}} \quad=$ Total depth (in $f t$ or $m$ ).

5. Now, from the $R_{m f}$ and $R_{m}$ values recorded on the log heading, determine the $\mathrm{R}_{\mathrm{mf}}$ and $\mathrm{R}_{\mathrm{m}}$ values at that particular formation temperature using the formula:

$R_{m f}$ at $T_{f}=R_{m f}$ at $T_{0}\left(T_{0}+C / T_{f}+C\right)$

Where $\mathrm{C}$ is the temperature offset.

$\mathrm{C}=6.8$ if imperial units are used and 21.5 if metric units are used.

$\mathrm{T}_{0}=$ Initial temperature at which $\mathrm{R}_{\mathrm{mf}}$ was first measured.

$\mathrm{R}_{\mathrm{m}}=$ Resistivity of mud, usually recorded on the log heading

6. Now read off SP amplitude from shale baseline to maximum constant deflection.

7. Determine bed thickness from SP deflection points.

8. Check whether the SP needs correction. If need be, correct for bed thickness, hole diameter, invasion and resistivity contrasts using the appropriate charts.
9. Now, knowing the formation temperature $\left(\mathrm{T}_{\mathrm{f}}\right)$, the static SP or SP (Corrected), recorded opposite a porous and permeable, non-shaly formation can be transformed into the resistivity ratio $R_{m} / R_{w e}$ in two ways: graphically as in figure 1 and by calculation.

\section{Graphically by use of chart:}

With the ratio $R_{m f e} / R_{w e}$ now determined and the resistivity $R_{m f}$ of a sample of mud filtrate measured, the equivalent formation resistivity, $\mathrm{R}_{\mathrm{we}}$, is easily calculated. However, the mud filtrate resistivity reported on the log heading or calculated at the formation temperature is its actual resistivity not its equivalent resistivity (Edwards et al 1963). To convert the measured mud filtrate resistivity $\left(\mathrm{R}_{\mathrm{mfe}}\right.$, the following rules are employed:

(a) For predominantly $\mathrm{NaCl}$ Muds.

i. If $\mathrm{R}_{\mathrm{mf}}$ at $75^{\circ} \mathrm{F}$ is greater than $0.1 \mathrm{ohm}-\mathrm{m}$, use $\mathrm{R}_{\mathrm{mf}} 0.85 \mathrm{ohm}-\mathrm{m}$ at Formation Temperature. This relationship is based on measurements made on many typical muds.

ii. If $\mathrm{R}_{\mathrm{mf}}$ at $75^{\circ} \mathrm{F}$ is less than $0.1 \mathrm{ohm}-\mathrm{m}$, use the $\mathrm{NaCl}$ (solid curves) in figure 2 to derive a value of $R_{m f e}$ from the measured $R_{m f e}$ value corrected to formation temperature.

(b) For fresh water or gypsum muds: the dashed curves of the chart in fig. 2 are used to convert $\mathrm{R}_{\mathrm{mf}}$ to $\mathrm{R}_{\mathrm{mfe}}$.

(c) Lime-based muds, despite their name, usually have negligible amounts of calcium and are treated as regular mud (see rule a).

(d) For predominantly $\mathrm{NaCl}$ Muds.

iii. If $\mathrm{R}_{\mathrm{mf}}$ at $75^{\circ} \mathrm{F}$ is greater than $0.1 \mathrm{ohm}-\mathrm{m}$, use $\mathrm{R}_{\mathrm{mf}} 0.85 \mathrm{ohm}-\mathrm{m}$ at Formation Temperature. This relationship is based on measurements made on many typical muds.

iv. If $\mathrm{R}_{\mathrm{mf}}$ at $75^{\circ} \mathrm{F}$ is less than $0.1 \mathrm{ohm}-\mathrm{m}$, use the $\mathrm{NaCl}$ (solid curves) in figure 2 to derive a value of $R_{m f e}$ from the measured $R_{m f e}$ value corrected to formation temperature.

(e) For fresh water or gypsum muds: the dashed curves of the chart in fig. 2 are used to convert $R_{m f}$ to $R_{m f e}$.

(f) Lime-based muds, despite their name, usually have negligible amounts of calcium and are treated as regular mud (see rule a). 

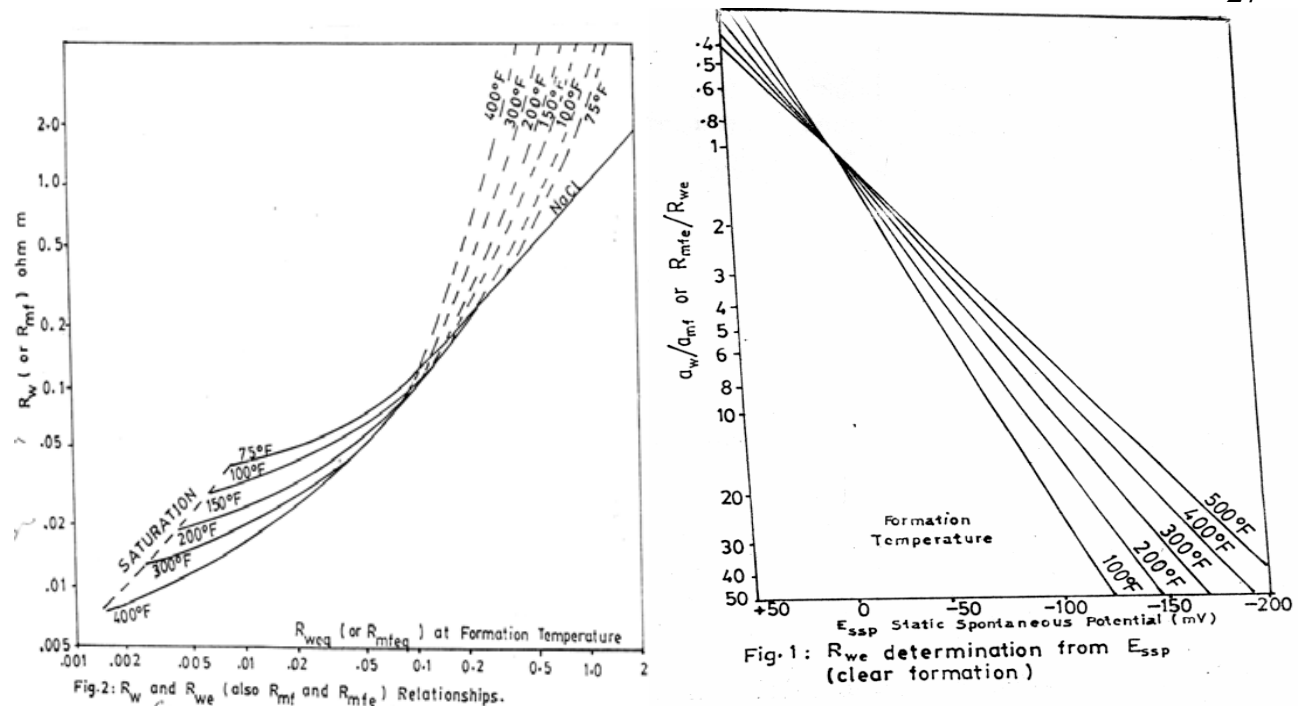

\section{By Calculation:}

(i)

The log units should be metric for the calculation to be done.

First determine the $\mathrm{R}_{\mathrm{mfe}}$

If $R_{m f}$ at $T_{f} \leq 0.1$, then $R_{m f e}=\left(1.46 R_{m f}\right.$ at $\left.T_{f}+77\right)$.

(iv)

If $R_{m f}$ at $T_{f}>0.1$, then $R_{m f e}=0.85 f t$ at $T_{f}$.

But

$$
\begin{aligned}
S S P & =K \log R_{m f e} / R_{w e}, \\
\frac{S S P}{K} & =\log R_{m f e} / R_{w e} \\
\therefore R_{m f e}=\frac{R_{m f e}}{R_{w e}} & =10^{(-S S P / K)}
\end{aligned}
$$

Lets denote $10^{(-\mathrm{SSP} / \mathrm{K})}$ as $\mathrm{R}_{\mathrm{sp}}$.

$\therefore \frac{R_{m f e}}{R_{w e}}=R_{s p}$

and $\therefore R_{w e}=\frac{R_{m f e}}{R_{s p}}$

10. (a) Determine $R_{w}$ from $R_{w e}$ value. The chart in Fig. 2 is also used to convert $R_{w e}$ to $R_{w}$. The solid curves, for very saline brine are derived from laboratory data on pure Nacl solutions. Thee solid curves are used for $R_{w e}$ and $R_{w}$ values less than $0.1 \mathrm{ohm}-\mathrm{m}$, they assume that in formation waters of this salinity $\mathrm{Nacl}$ is the dominant salt. (b) By simple calculation as follows:

i. If $R_{w e}>0.12$, then $R_{w}$ at $T_{f}$ $\left(0.58-\left[\left(6.9 R_{w e}+2.4\right)\right]\right.$

ii. If $\mathrm{R}_{\mathrm{we}} \leq 0.12$, then $\mathrm{R}_{\mathrm{w}}$ at $\mathrm{Tf}$

$\left(77 R_{w e}+5\right) / 146-337 R_{w e}$

11 Check $R_{w}$ from Sp against another source.

\section{PRECAUTION AND CONCLUSION}

The static SP value can only be obtained directly from the SP curve, if the bed is clean thick, porous and only moderately invaded; and if the formation is saline and the drilling mud is not too reactive. These conditions are not always met. When 
they are not, the recorded SP deflection (in milivolts) must be corrected to a static SP value for bed thickness, hole diameter, invasion and resistivity contrasts (Pirson, 1963, Frick 1962).

It is assumed that the recorded SP curve seldom contains an electrokinetic potential component. Although this is generally the case very low permeability formation, depleted pressure formation, or the use of very heavy drilling mud give rise to a significant electrokinetic potential. In these cases, an $R_{w}$ derived from the SP curve will probably be too low, so other sources of $R_{w}$ data should be explored (Tixer et al, 1965). Knowledge of $R_{w}$ values is invaluable. It opens the lock to some other important parameters in formation evaluation. Rw is useful in calculating water saturation in the formula

$$
S_{w}=F R \cdot R_{w} / R_{t} .
$$

When water saturation is known, then hydrocarbon in place, $\mathrm{HC}=\left(1-S_{w}\right)$, is derivable. And since qualification on hydrocarbons and calculation of reserves is indispensable in production, formation water resistivity, $R_{w}$, remains one of the most important interpretational parameters in well log analysis.

\section{REFERENCES}

Edwards, D.P., Lacour-Gayet, P.J. and Suan, J., 1963. Log Evaluation in Wells drilled with inverted oil emulsion mud SPE 1020y, San Anthonio. pp.313-318.

Frick, T.C. 1962. Petroleum Production Handbook, McGraw-Hill, New York. pp.19-22.

Pirson, S.J., 1963. Handbook or Well Log Analysis. Prentice-Hall Inc. pp.42 - 43.

Schlumberger, 1989. Log Interpretation principles. pp.8-9.

Smolen, J.J. 1977. Formation Evaluation Using Wireline Formation Tester Pressure Data SPE 6822, Denver. pp. 4 - 8 .

Tixer, M. P.; Alger, R.P. and Tanguy, D.R. 1965. New developments in Induction and sonic Logging. SPE 1 300-G Dallas. 